\title{
Urea transporter mRNA in papilla and pelvic epithelium of kidney in normal and low protein fed sheep*
}

\author{
N. Artagaveytia ${ }^{1}$, J.-M. Elalouf ${ }^{2}$, R. Boivin ${ }^{3}$, \\ C. de Rouffignac ${ }^{2}$ and A. Cirio ${ }^{4}$
}

\begin{abstract}
${ }^{1}$ Departamento Básico de Medicina, Facultad de Medicina, 11800 Montevideo, Uruguay ${ }^{2}$ Service de Biochimie et de Génétique Moléculaire, CEA /Saclay, 91191 Gif-Sur-Yvette, France ${ }^{3}$ UMR Laboratoire de Physiologie, Ecole Vétérinaire de Lyon, 69280 Marcy L'Etoile, France ${ }^{4}$ Departamento de Fisiología, Facultad de Veterinaria, 11600 Montevideo, Uruguay
\end{abstract}

\begin{abstract}
An urea transporter (UT) cDNA fragment, sharing high identity with rabbit, rat and human UTA2, was cloned by RT-PCR from papilla and upper pelvic epithelium of sheep kidney. Quantitative RT-PCR after low protein diets disclosed, compared to normal protein fed sheep, a significant reduction in UT mRNA levels in pelvic epithelium ( $852 \pm 172$ vs $2024 \pm 260$ molecules; $\mathrm{P}<0.01$ ) and a tendency to its increase in papilla ( $7959 \pm 1741$ vs $5447 \pm 1040$ molecules; NS). The reduction of UT expression in the upper pelvic epithelium could be relevant for the renal urea conservation in protein-restricted sheep.
\end{abstract}

KEY WORDS: urea transporter mRNA, kidney, low protein diet, sheep

\section{INTRODUCTION}

The amount and distribution of the renal urea transporters (UT) varied among species. One reason for this variability is thought to be the dietary protein supply, the abundance of UT reflecting the adaptation (nitrogen salvaging) to differences in dietary protein, herbivorous having more diluted protein intake than omnivorous (Hediger et al., 1996). The advantage of this adaptation should be relevant in ruminants in which endogenous urea serves as nitrogen source for protein synthesis in their forestomachs. In low protein fed sheep, urea conservation is achieved by

\footnotetext{
* Supported by the CEA and the INRA (France), the Universidad de la República (Uruguay) and by the Grant U94 B06 from ECOS France-Uruguay

${ }^{4}$ Corresponding author: e-mail: cirio@montevideo.com.uy
} 
a reduction in filtered load of urea and an enhanced urea reabsorption from renal tubules and pelvis (Cirio and Boivin, 1990). Characterization of UT-A mRNAs in urea-transporting epithelia of the sheep kidney is lacking. The aims of this work were a. the identification and cloning of a cDNA encoding an urea transporter in papilla and upper pelvic epithelium of sheep kidney and $b$. the comparison of the expression of urea transporter mRNAs in these structures in normal and low protein fed sheep.

\section{MATERIAL AND METHODS}

Three grazing (aim a) and six caged (aim b) ewes were used. The last were divided into two groups $(\mathrm{n}=3)$ and fed once daily with normal (NP group) and low protein (LP group) diets (67 and $42 \mathrm{~g}$ crude protein daily intake, respectively) during five weeks. Renal functional studies (RPF, GFR and clearance, filtered load and fractional excretion of urea) were performed using standard clearance methods. Experiments were carried out in accordance with international ethical guidelines.

For renal tissues sampling, kidneys were isolated under general anaesthesia, sectioned longitudinally exposing the crest-type papilla and the pelvic space with its projections reaching the outer zone of the medulla and giving rise to specialized fornices. Pelvic epithelium was microdissected at the outer zone of the medulla, where urea transfer is expected to be maximal. Sampling places are shown on Figure 1.

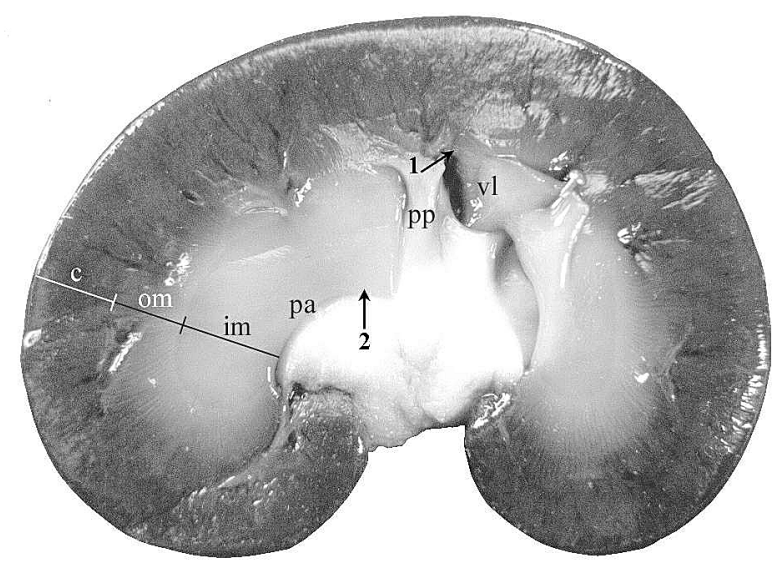

Figure 1. Sagital cut of the sheep kidney. The inner medulla has been cut, folded and fixed with a pin, discovering one pelvic projection ( $\mathrm{pp}$ ) that, enfolding the blood vessels, reaches deep into the outer medulla and gives rise to leaf-like processes called specialized fornices (not shown). Tissue samples were obtained from the visceral leaf (vl) of the pelvic epithelium lining the outer medulla at the fornix region (arrow 1) and from the crest-type papilla (pa, arrow 2). c - cortex, om - outer medulla, im - inner medulla 
Total RNA from kidney tissues was isolated as described by Chomczynski and Sacchi (1987). For cloning of an ovine UT-A cDNA, RT-PCR was performed on 1 $\mu \mathrm{g}$ of total RNA extracted from five independent papillary samples, using degenerate primers (Table 1). PCR products (770 bp) were pooled and cloned into the EcoRV site of pBluescript vector (BSSK+). Quantification of UT-A mRNAs expression was performed by RT-PCR on 3 ng of total RNA from kidney tissues, using primers designed from the cloned ovine cDNA and with the addition of 2000 molecules of UT-A mutant cRNA as an internal standard (Table 1). To generate the mutant cRNA by in vitro transcription, a Pvu II restriction site at position 519 in the cloned ovine UTA cDNA was introduced. To discriminate wild type and mutant fragments, RT-PCR products (363 bp) were digested with Pvu II (5 Units) and after electrophoresis the gel was exposed to a storage phosphor screen and analysed using scanner software. The number of mRNA molecules in pelvic epithelium and papilla was calculated from the ratio of the signals obtained for wild type and mutant RNAs.

Table 1. Oligonucleotides, primers and PCR protocols. Primers were designed using the Oligo software (MedProbe, Oslo, Norway)

\begin{tabular}{llr}
\hline Application & \multicolumn{1}{c}{ Sequence / Protocol } & Position \\
\hline \multirow{3}{*}{ PCR-cloning } & Degenerated UT-A & \\
& 5'-GTNCARAAYCCNTGGTGGCG-3' & 256 \\
& 5'-TARCARAANGGCCANGTRCA-3' & 1028 \\
& 35 cycles (95 ${ }^{\circ} \mathrm{C}-30 \mathrm{~s}, 58^{\circ} \mathrm{C}-1 \mathrm{~min}$, 72 $\left.^{\circ} \mathrm{C}-1.5 \mathrm{~min}\right)$; 10min elong. & \\
RT-PCR & Ovine UT-A & 311 \\
& 5'-CCACGGGCCACTACAACCTCTTCT-3' & 674 \\
& 5'-GGAGGTGCGTCTGCCAGGTGAT-3' & \\
\hline
\end{tabular}

\section{RESULTS}

The expected $770 \mathrm{bp}$ PCR fragment was found in all papillary samples tested as in pelvic epithelium ones. The deduced amino-acid sequence shared 92 and $93 \%$ identity with UT-A2 protein from rabbit and rat, and from human, respectively. This sequence analysis therefore indicates that we have cloned a cDNA corresponding to part of sheep UT-A (GenBank accession number $=$ AY303685).

LP ewes showed a sparing pattern in its urea tubular handling (significant reductions in RPF, GFR and the filtered load, the fractional excretion and the urine elimination of urea) compared to NP animals. UT-A mRNA expression was significantly lower in pelvic epithelium than in papillary tissue in NP ewes (2024 \pm 260 vs $5447 \pm 1040$ molecules; $\mathrm{P}<0.05$ Mann Whitney test). LP diet increased this difference to a factor of $10(\mathrm{P}<0.005)$, by means of a significant reduction of UT-A mRNA expression in pelvic epithelium and a tendency to its 
increase in papillary tissue $(852 \pm 172, \mathrm{P}<0.01$ and $7959 \pm 1741$ molecules, NS, respectively, compared to NP diet).

\section{DISCUSSION}

The LP-induced decrease in UT expression in pelvic epithelium at the outer medulla level could play a role in the renal conservation of urea. Indeed, LP diets reverse the normal medullary urea gradient, shifting its highest concentration to the outer medulla and creating a steep urea concentration gradient from the outer medulla to the final urine filling the fornices of the upper pelvic space (reviewed in SchmidtNielsen, 1990). Since the extremely thin pelvic epithelium at this level allows the exchange of urea between pelvic urine and tissue (Cirio and Boivin, 1990; SchmidtNielsen, 1990), a decrease in urea permeability due to a lesser expression of the UTs could be relevant for urea conservation, reducing its loss from the outer medulla.

If the inner medulla of the sheep expresses UT-A1 and -A2 isoforms, the two major products of the UT-A gene, these isoforms were co-amplified in our study. In rats, both isoforms seem to be independently regulated and respond differently to changes in protein content (Smith et al., 1995) and in duration (Ashkar et al., 1995) of experimental diets. It is therefore hazardous, in our case, to interpret the data observed for papilla samples. Further studies are necessary to distinguish between UT-A1 and -A2 isoforms and to separately analyse its LP diet induced abundance.

\section{CONCLUSIONS}

A cDNA, corresponding to the UT-A isoform, was cloned from papilla and pelvic epithelium of sheep kidney. LP diet had a differential effect on UT-A mRNA expression in both tissues. Its reduction in the upper pelvic epithelium could be relevant for urea conservation in protein-restricted sheep.

\section{REFERENCES}

Ashkar Z.M., Martial S., Isozaki T., Price S.R., Sands J.M., 1995. Urea transport in initial IMCD of rats fed a low-protein diet : functional properties and mRNA abundance. Amer. J. Physiol. 268, F1218-1223

Chomczynski P., Sacchi N., 1987. Single step method of RNA isolation by acid guanidinium thyocianate-phenol-chloroform extraction. Anal. Biochem. 162, 156-159

Cirio A., Boivin R., 1990. Urea recycling from the renal pelvis in sheep: a study with $\left[{ }^{14} \mathrm{C}\right]$ urea. Amer. J. Physiol. 258, F1196-1202

Hediger M.A., Smith C.P., You G., Lee W.S., Kanai Y., Shayakul C., 1996. Structure, regulation and physiological roles of urea transporters. Kidney Int. 49, 1615-1623

Schmidt-Nielsen B., 1990. Function of the pelvis. In: Urinary Concentrating Mechanisms. Karger, Comp. Physiol., Basel, pp. 103-140

Smith C.P., Lee W.S., Martial S., Knepper M.A., You G., Sands J.M., Hediger M.A., 1995. Cloning and regulation of expression of the rat kidney urea transporter (rUT2). J. Clin. Invest. 96, 1556-1563 